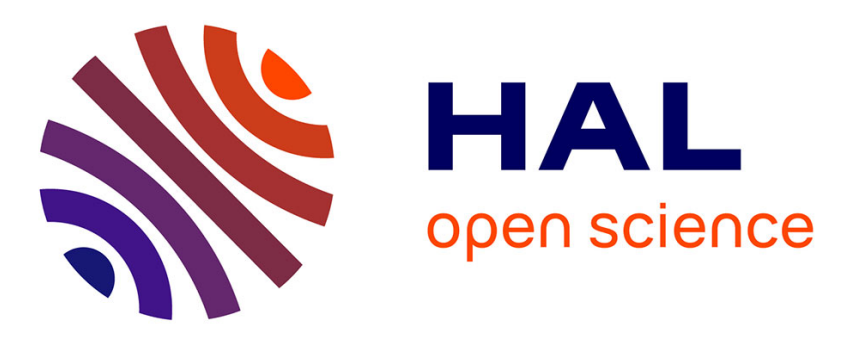

\title{
Articulation des niveaux microscopiques et mésoscopiques dans les analyses de pratiques de classe à partir de vidéos
}

Andrée Tiberghien, Patrice Venturini

\section{- To cite this version:}

Andrée Tiberghien, Patrice Venturini. Articulation des niveaux microscopiques et mésoscopiques dans les analyses de pratiques de classe à partir de vidéos. RDST - Recherches en didactique des sciences et des technologies, 2015, Les cadres théoriques et méthodologiques, 11, pp.53-78. halshs-01216999

\section{HAL Id: halshs-01216999 \\ https://shs.hal.science/halshs-01216999}

Submitted on 21 Nov 2016

HAL is a multi-disciplinary open access archive for the deposit and dissemination of scientific research documents, whether they are published or not. The documents may come from teaching and research institutions in France or abroad, or from public or private research centers.
L'archive ouverte pluridisciplinaire HAL, est destinée au dépôt et à la diffusion de documents scientifiques de niveau recherche, publiés ou non, émanant des établissements d'enseignement et de recherche français ou étrangers, des laboratoires publics ou privés. 


\title{
Articulation des niveaux microscopiques et mésoscopiques dans les analyses de pratiques de classe à partir de vidéos
}

\author{
Andrée Tiberghien \\ CNRS et université de Lyon, UMR ICAR \\ Patrice Venturini \\ Université de Toulouse Jean-Jaurès, UMR EFTS
}

RÉSUMÉ • Cette étude d'ordre méthodologique porte sur le développement d'une analyse à l'échelle microscopique à partir d'enregistrements vidéo de classes de physique en articulation avec une analyse à l'échelle mésoscopique. L'échelle microscopique correspond à quelques secondes et celle mésoscopique à des moments d'une séance de l'ordre de quelques minutes ou dizaines de minutes. Le choix d'une orientation théorique pragmatique de la théorie de l'action conjointe en didactique a été pris. En référence aux travaux sur les actes de langage, au niveau microscopique, l'acte a été choisi comme l'unité d'analyse ; il est caractérisé par trois dimensions : pragmatique, interactionnelle et représentationnelle. L'épisode est une unité intermédiaire entre les niveaux microscopique et mésoscopique. Le jeu est l'unité mésoscopique; il est caractérisé par trois types d'évolutions, ceux du savoir, de la responsabilité vis-à-vis du savoir des élèves et du professeur, et du milieu. Nous avons ainsi construit un ensemble cohérent d'analyses des pratiques de classe aux niveaux microscopique et mésoscopique opératoires pour différentes situations de classe, celle d'introduction en classe entière d'une démarche d'investigation, ou celle d'un travail en petits groupes entre élèves et avec l'intervention du professeur.

MOTS-CLÉS • didactique, physique, méthodologie, acte de communication, échelle

ABSTRACT • Connecting microscopic and mesoscopic levels in the analyses of classroom practices from videos

This methodological study is focused on the development of a microscopic analysis from video recordings of physics classes in relation to an analysis at mesoscopic scale. The microscopic scale corresponds to a few seconds and the mesoscopic one to moments of a 
teaching session of the order of minutes or dozens of minutes. The choice of a pragmatic theoretical orientation of the theory of joint action in didactics was taken. With reference to works on speech acts, at the microscopic level, the act was chosen as the unit of analysis; it is characterized by three dimensions: pragmatic, interactional and representational. The episode is an intermediate unit between the microscopic and mesoscopic levels. The game is the mesoscopic unit; it is characterized by three types of evolution, those of knowledge, of the students and teacher responsibility vis-à-vis knowledge, and the environment. We have built a coherent set of analyses of classroom practices at microscopic and mesoscopic levels operating for different classroom situations, like the introduction of an inquiry session in whole class, or a small group work between students and with the teacher intervention. KEYWORDS • didactics, physics, methodology, communication act, scale

\section{Introduction et problématique}

De l'avis de nombreux chercheurs, l'étude du fonctionnement des classes, que les données vidéo permettent de développer, est complexe. En effet, même si les vidéos ne donnent à voir qu'une partie des pratiques d'enseignement et d'étude, l'analyse de ce qui est donné à voir pose de nombreuses questions théoriques et méthodologiques. Certaines ont été traitées, au moins en partie, dans des travaux précédents (par exemple Mortimer \& Scott, 2003; Scott et al., 2005; Tiberghien et al., 2007; Tiberghien, Malkoun \& Seck, 2008; Venturini \& Tiberghien, 2012).

L'une d'entre elles est liée à la variété des échelles de temps auxquelles se déroulent les différents événements ou processus qui constituent ces pratiques (Lemke, 2001). L'étude menée en 2012 (Venturini \& Tiberghien, 2012) sur l'étude des pratiques de classe dans le cas de démarche d'investigation nous a en effet conduits à soulever la question des liens entre échelles. Cette étude a porté sur l'analyse de pratiques de classe développées au cours d'une séance concernant la mise en œuvre d'une démarche d'investigation en physique. Pour cette analyse, nous avons structuré la séance au niveau mésoscopique en thèmes, selon l'organisation sociale de la classe et aussi en jeux didactiques, en référence à la théorie de l'action conjointe en didactique (TACD) comme nous le présentons ci-dessous. Pour caractériser les jeux didactiques, nous les avons découpés en épisodes de l'ordre d'une à deux minutes que nous avons analysés en utilisant les mêmes descripteurs que ceux utilisés au niveau mésoscopique, et notamment ceux fournis par la TACD. Le changement d'épisode était déterminé par le changement de la modalité d'au moins un de ces descripteurs. Toutefois, dans la communication de notre étude, nous n'avons argumenté ni la légitimité de ce choix de découpage des jeux (niveau mésoscopique) en épisodes, ni la légitimité des modalités mésoscopiques utilisées pour caractériser ces derniers. Nous n’avons pas plus explicité les liens entre la caractérisation des épisodes et les 
caractéristiques d'événements spécifiques beaucoup plus courts (de l'ordre de la seconde), constitutifs des épisodes. Ceci conduit à poser la question de la contribution des observables au niveau microscopique à la caractérisation mésoscopique ; en conséquence la question de comment caractériser les événements au niveau microscopique se pose. Dans cette perspective, quel est alors le statut de l'épisode, sorte d'échelon intermédiaire entre le niveau microscopique et le niveau mésoscopique?

L'étude que nous présentons ici a l'ambition de traiter au moins en partie ces questions, en considérant qu'il est utile de s'appuyer sur des événements microscopiques. Elle présente les choix théoriques et méthodologiques que nous avons faits pour qu'une telle analyse au niveau microscopique permette de documenter celle menée au niveau mésoscopique, dans le cas particulier où ce niveau est analysé notamment à l'aide de la théorie de l'action conjointe. Ceci suppose nécessairement que l'analyse mésoscopique informe aussi le niveau microscopique. Pour illustrer nos propos, nous donnerons deux exemples fondés sur deux extraits d'études que nous avons menées.

\section{Cadre théorique}

Nous présentons tout d'abord succinctement notre approche au niveau mésoscopique dans laquelle la théorie de l'action conjointe en didactique (TACD) joue une place prépondérante (Sensevy, 2011). Nous développons ensuite nos choix pour une l'analyse au niveau microscopique et enfin nous proposons une manière d'articuler l'un à l'autre à travers un niveau (échelon) intermédiaire fondée sur des narrations didactiques.

\section{1. Éléments théoriques au niveau mésoscopique}

La théorie de l'action conjointe en didactique s'inscrit dans le paradigme de l'action conjointe en sciences sociales : l'action humaine est sociale, elle est pensée comme une action conjointe et prend nécessairement en compte l'action de l'autre. Dans le cas de l'action didactique, l'enseignement et l'apprentissage sont ainsi considérés comme des actions nécessairement conjointes Sensevy (2011).

Ceci suppose donc un certain ajustement mutuel :

"Agir dans le monde social, c'est agir conjointement, et agir conjointement, c'est s'ajuster à autrui. Pour Mead et Blumer, cette assertion concerne le monde social en général. Le terme transaction entre en résonance forte avec cette caractérisation. Une transaction, comme le montre Vernant (1997) suppose une relation particulière à la fois à "l'environnement", au milieu (aspect "mondain" de la transaction) et à autrui (aspect "intersubjectif " de la relation). [...] "transagir", c'est agir à travers (trans) autrui et à travers "l'environnement". À travers, ici, signifie aussi à la fois au moyen de, grâce à, comme dans l'expression "à travers l'étude de" ". (ibid., p. 52-53)

À partir de ces idées de base, le cadre théorique de la TACD propose la modélisation de la relation didactique en termes de jeu en se référant au jeu de langage 
de Wittgenstein et au jeu social de Bourdieu. Le jeu didactique implique le professeur, l'élève et le savoir dont il est l'enjeu; il permet une approche systémique. En d'autres termes le jeu didactique nous permet d'aborder de manière holistique les actions relevant de l'enseignement et de l'apprentissage. Un jeu n'est gagnant pour le professeur que si l'élève (ou les élèves) est (sont) gagnant(s) dans la mesure où la construction du savoir ne peut être faite que par l'élève. Le jeu est considéré dans une perspective ternaire, le savoir étant l'objet d'une transaction entre professeur et élève(s). La classe, étudiée du point de vue de l'action didactique, est alors modélisée comme un ensemble de jeux didactiques, chacun ayant un enjeu particulier lié à la construction de savoirs. Cette modélisation en jeux permet d'introduire les deux concepts au cœur de la TACD, le contrat et le milieu. Le contrat recouvre le système d'attentes réciproques entre le professeur et les élèves qui structure l'action didactique et plus largement les systèmes stratégiques utilisés par le professeur et l'élève pour traiter du problème en jeu dans la transaction et donc jouer le jeu didactique correspondant (Sensevy, 2013). Le milieu est constitué par les éléments de l'environnement matériel et symbolique pris en compte par le professeur ou l'élève dans la transaction; généralement, il n'est pas identique pour le professeur et pour l'élève. De plus, quatre types d'actions caractérisent la façon dont le professeur joue et fait jouer le jeu didactique : définir le jeu, tenter de le dévoluer aux élèves qui doivent accepter la responsabilité de le jouer et donc d'essayer de le gagner; réguler son développement de manière à ce que les élèves adaptent leur manière de jouer afin de gagner; enfin institutionnaliser le résultat du jeu.

Le développement du jeu et donc les dynamiques à l'œurre dans le déroulement de la transaction sont décrits au travers de la formalisation de trois genèses ou encore trois évolutions temporelles : celle du savoir dans la classe (chronogenèse), celle de la responsabilité du professeur et des élèves vis-à-vis du savoir (topogenèse), et enfin celle de l'environnement qui joue un rôle dans les actions du professeur et des élèves (mésogenèse). Ces genèses contribuent à la caractérisation du contrat et du milieu pour chaque jeu.

Ainsi, l'échelle temporelle du jeu est en lien avec le rythme d'évolution de l'action didactique au sein de la classe. En effet, le jeu change dans la mesure où l'objet et les formes de transaction sont modifiés, ce dont les genèses et leurs discontinuités rendent compte. Ces changements de jeu interviennent sur des périodes allant de quelques minutes à quelques dizaines de minutes, durées qui caractérisent le niveau mésoscopique. La question de la caractérisation à l'échelle microscopique se pose différemment dans la mesure où les catégories de chrono, méso et topo genèses ne sont pas adaptées pour cette échelle de temps. En effet, ces catégories nécessitent le plus souvent une vision temporelle suffisamment importante pour identifier une évolution. Aussi avons-nous été amenés à construire d'autres descripteurs et pour cela à faire des choix liés à l'échelle microscopique que nous présentons ci-dessous. 


\section{2. Éléments théoriques au niveau microscopique}

De manière générale, les études didactiques qui utilisent les échelles temporelles en distinguent trois, une échelle au niveau microscopique de l'ordre de la seconde, une échelle au niveau macroscopique correspondant à la durée du corpus à savoir d'une à plusieurs séances, et une échelle intermédiaire appelée mésoscopique allant de quelques minutes à quelques dizaines de minutes (Tiberghien et al., 2007). Notons que nous utilisons le terme d'échelle temporelle, mais qu'en fait nous suivons Wortham (2006) : "I use Lemke's "timescale" terminology because I find it useful, but I acknowledge that each relevant process has both spatial and temporal extension. When I discuss the "local timescale," I mean a spatiotemporal niche bounded spatially by [...] (the) classroom and temporally by the academic year in question. " "

Avec cette perspective, nous présentons notre nouvelle approche au niveau microscopique.

\subsubsection{Choix qui fondent notre approche à l'échelle microscopique}

À cette échelle de temps, il est nécessaire de s'orienter vers des catégories adaptées à des événements ${ }^{2}$ de l'ordre de la seconde et cohérentes avec le point de vue pragmatique et actionnel de la TACD. Ces catégories doivent être opérationnelles pour analyser les enregistrements vidéo de classe et leur transcription.

Notre approche reprend l'idée que l'action didactique est "organiquement communicationnelle". (Sensevy, 2011, p. 98). Ceci nous conduit à nous tourner vers la communauté des sciences du langage qui prend une orientation pragmatique pour étudier la communication.

L'approche de l'action conjointe rejoint le nouage que fait Charaudeau (2004) entre action et communication. Pour lui, le but de l'action et la visée de la communication sont complètement imbriqués; ainsi quand un élève demande à son binôme de décider ensemble de la réponse, il s'agit "d'un projet d'action (fournir une réponse) et d'une communication dont la visée cherche à modifier le comportement des autres" (ibid., p. 161). Cette approche, qui vise à concevoir les rapports entre action et langage, nous permet d'étudier le niveau microscopique de l'action conjointe en distinguant plusieurs dimensions sans pour autant les interpréter indépendamment les unes des autres. Pour Charaudeau, trois conceptions articulent

1 J'emprunte à Lemke la terminologie "échelle de temps" parce que je la trouve utile, mais je reconnais que chaque processus pertinent a à la fois une étendue spatiale et temporelle. Quand je discute de "l'échelle de temps locale", je veux dire une niche spatio-temporelle délimitée spatialement par [...] (la) classe et temporellement par l'année académique en question.

2 Ici événement est proche du sens physique du terme, quelque chose qui peut être observé et être repéré dans l'espace et dans le temps; seuls des événements pertinents du point de vue de la théorie seront retenus, c'est-à-dire ceux qui peuvent donner du sens à l'étude des pratiques de la classe observée. Par exemple la couleur du vêtement porté par l'enseignant ne sera prise en compte que si cela prend sens dans les interactions, au vu des concepts constituant la théorie. 
ou encore nouent action et langage : "la représentationnelle, la pragmatique et l'interactionnelle" (p. 161). Ces trois conceptions constituent les trois dimensions du modèle microscopique que nous avons construit en cohérence avec le point de vue de l'action qui sous-tend la TACD. Ces trois dimensions caractérisent un acte aussi bien verbal que non verbal comme par exemple lever le doigt pour demander la parole ou brancher un circuit.

Ainsi, pour rendre opératoire l'analyse au niveau microscopique nous empruntons et importons une théorie linguistique. Nous pensons que cet emprunt est compatible avec la TACD, car le nouage entre action et communication partage une vue pragmatique commune même si nous sommes bien conscients de la nécessaire adaptation de cette approche linguistique pour notre analyse.

\subsubsection{Caractérisation des actes verbaux et non verbaux du niveau microscopique selon trois dimensions}

L'acte constitue notre unité d'analyse microscopique et ce choix nécessite de préciser la différence entre action et acte. Si l'empan de l'action didactique est très variable (Fillietaz, 2004), celle-ci incluant plusieurs transactions successives, celui de l'acte que nous considérons comme le plus petit événement gardant un sens du point de vue de la transaction didactique, est de l'ordre de la seconde.

Ainsi, le cas de l'élève qui lève le doigt pour demander la parole est un acte significatif dans la transaction professeur - élève; par contre si on le décompose en éléments du type "l'élève lève le bras" puis "pointe son doigt vers le haut", la décomposition n'est pas pertinente pour notre analyse car le sens de chacun de ces actes ne peut être construit dans la perspective de la transaction en cours. Ce point de vue laisse une grande part à l'interprétation et n'enlève pas la difficulté du découpage de la transaction en actes. Comme le souligne Kerbrat-Orecchioni (1995), le problème du découpage est abandonné à l'intuition de l'analyste dans la mesure où les définitions ne donnent pas de critères opératoires ${ }^{3}$ et qu'en plus "différents actes peuvent non seulement se succéder dans un même énoncé, mais aussi s'y amalgamer" (p. 7). Le problème est similaire pour les actes non verbaux. Précisons avant de poursuivre que si nous avons cherché à découper les transactions acte par acte, il arrive que certains actes soient superposés ou même amalgamés.

\subsubsection{La dimension pragmatique}

Cette dimension est reliée aux forces illocutoire et perlocutoire du langage et rattachée à l'intentionnalité du professeur ou des élèves, en référence aux nombreux travaux sur les actes de langage (voir par exemple Searle, 1969; Searle \& Vanderveken, 1985). Cependant comme l'analyse très clairement Kerbrat-Orecchioni (1995), la

3 "On ne peut en effet guère tirer parti de définitions telles que : "l'acte de langage est le segment discursif associé à un seul contenu propositionnel" (Moeschler, 1985, p. 81), ou encore : "l'acte est une unité discursive qui véhicule une intention communicative distincte" (Heddescheimer \& Roussel, 1986, p. 48)." (Kerbrat-Orecchioni, 1995, p. 7). 
multiplicité des taxinomies d'actes de langage nous a conduits à choisir nos propres catégories, établies de manière inductive et adaptées à notre propos qui est d'étudier l'évolution du savoir dans la classe.

Nous avons ainsi identifié des actes que nous avons regroupés sous des désignations génériques elles-mêmes classées en trois catégories. La première met en jeu des actes introduisant de nouvelles idées en considérant le point de vue du rapport aux objets de savoir. L'autre catégorie suppose qu'une proposition ait déjà été faite. Enfin la dernière catégorie est relative à la gestion de la communication (Le tableau 1 donne l'essentiel de ces catégories); cette liste d'actes est non exhaustive.

Pour chacun de ces actes, nous précisons aussi dans l'analyse l'intentionnalité correspondante de l'acteur lorsqu'elle n'est pas explicite et qu'il est possible de l'inférer.

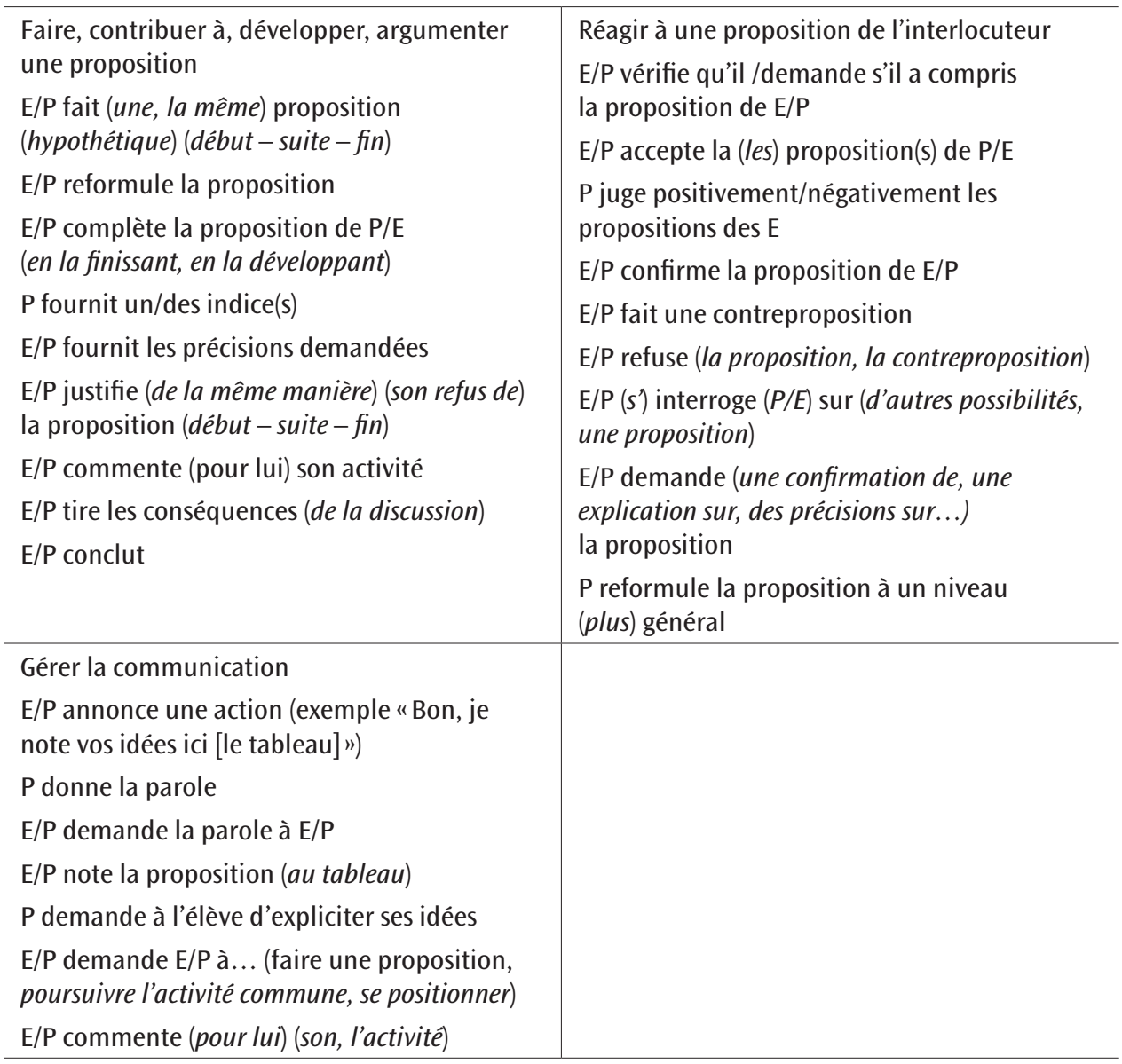

Tableau 1 : actes apparaissant dans des séances d'enseignement de la physique

$E$ désigne un ou des élèves. $P$ désigne le professeur. Les termes entre parenthèses varient selon le contexte et spécifient l'acte. Les catégories et les spécifications ne sont pas exhaustives, elles s'enrichissent aussi au cours des analyses. La catégorisation des actes est uniquement un moyen de les présenter et de les retrouver rapidement au cours de l'analyse. II ne s'agit pas pour nous d'établir une taxonomie. 


\subsubsection{La dimension interactionnelle}

A l'école, un acte de langage contribue généralement à un discours interactif, mais il peut arriver qu'il participe à un discours non-interactif au sens de Mortimer et Scott (2000).

Lorsqu'il s'agit d'un discours interactif, les actes de langage s'insèrent généralement dans le pattern "Initiation - Réponse - Poursuite ${ }^{4}$ (IRP) identifié par Sinclair et Coulthard $(1975,1992)$ à partir de leurs analyses structurales des discours de classe. Si cette structure est typique des échanges dirigés par l'enseignant, nous l'avons adaptée dans nos analyses afin de caractériser les échanges entre élèves dans lesquels le professeur est peu ou pas présent. Dans ce cas toutefois, la progression des échanges n'est pas aussi ordonnée que lorsque c'est le professeur qui la dirige. Une séquence IRP peut être interrompue momentanément, laisser la place à d'autres échanges puis reprendre, si bien que ces séquences sont souvent enchevêtrées.

Pour ce qui est de l'initiation et de la réponse associée : Sinclair et Coulthard (1992, p. 16) considèrent que "there are three major acts which probably occur in all forms of spoken discourse - elicitation, directive and informative - and they appear in classroom discourse as the heads of Initiation moves ${ }^{5}$ ". Nous distinguons donc trois types d'initiations, faire parler (questions), faire faire (injonctions) et donner de l'information pour faire penser (propositions argumentées ou non). Si les formes de réponses aux injonctions et propositions sont très liées au contenu de ces dernières, dans une classe les questions appellent souvent des réponses orales. Le couple question - réponse peut alors être caractérisé selon la nature de la question et selon la longueur et le niveau de raisonnement exigé par la réponse quelle que soit la personne qui initie l'échange, professeur ou élève (Graesser \& Person, 1994). Ainsi, les questions fermées (avec ou sans indices) invitent à des réponses courtes qui contribuent de manière limitée au thème en cours. Au contraire, les questions ouvertes (avec ou sans indices) amènent généralement des réponses plus longues, exigeant un raisonnement plus approfondi, et celles-ci contribuent de manière beaucoup plus étendue au thème en jeu ${ }^{6}$. Mais les questions peuvent aussi simplement viser une relance des échanges ou inviter à un positionnement et les réponses peuvent aussi être étrangères au thème en jeu, voire avoir uniquement une fonction sociale comme chercher à maintenir l'échange (par exemple au sein d'un groupe d'élèves).

Pour ce qui est de la poursuite: Chin (2006) a identifié plusieurs types pour le

4 Ce pattern est plus connu sous l'acronyme IRF pour Initiation - Response - Follow-up.

5 "Il y a trois actes majeurs qui probablement apparaissent dans toutes les formes de discours parlé - élicitation (incitation), directive (consigne) et information - et dans le discours de classe ils sont liés à l'apparition d'une nouvelle Initiation".

6 Par exemple, la réponse peut impliquer un raisonnement logique, un raisonnement causal, ou encore un raisonnement orienté vers un but particulier, chacun de ces types de raisonnement étant lié aux niveaux les plus élevés de la taxonomie de Bloom (Bloom et al., 1956). 
dernier constituant du pattern isolé par Sinclair et Coulthard. D'après ses analyses, il peut s'agir d'une répartie totalement neutre vis-à-vis des savoirs en jeu, d'une évaluation de la réponse, d'une indication orientant les élèves dans une direction particulière, d'une déclaration finale clôturant les échanges, ou d'une déclaration intermédiaire visant à stabiliser au moins provisoirement certains éléments.

Enfin, lorsque les actes sont les constituants d'une séquence non-interactive (qui peut ou non être dialogique), il est aussi possible de les caractériser. Ils peuvent alors concerner par exemple des déclarations liées aux savoirs, des injonctions liées à la gestion de la classe, etc.

Le tableau 2 récapitule l'ensemble de ces descripteurs.

\begin{tabular}{|c|c|c|}
\hline \multicolumn{3}{|l|}{ Discours interactif } \\
\hline Initiation & Réponse & Poursuite \\
\hline Injonction & Contribution limitée au thème & Évaluation de la réponse \\
\hline Question fermée & en jeu & Orientation de l'élève dans \\
\hline avec/sans indice & Contribution étendue au thème & une direction particulière \\
\hline Question fermée & en jeu & Répartie neutre vis-à-vis \\
\hline & еріен & \\
\hline Question de relance & & Déclaration finale clôturant \\
\hline Question invitant à un & Maintien de l'échange & les échanges \\
\hline positionnement & & Déclaration intermédiaire \\
\hline Question autre & & stabilisant certains éléments \\
\hline Proposition argumentée & & \\
\hline Proposition non argumentée & & \\
\hline \multicolumn{3}{|l|}{ Discours non interactif } \\
\hline Déclaration liée aux savoirs & $\begin{array}{l}\text { Injonction liée à la gestion } \\
\text { de la classe }\end{array}$ & $\cdots$ \\
\hline
\end{tabular}

Tableau 2 : descripteurs utilisés pour caractériser la dimension interactionnelle des actes

\subsubsection{La dimension représentationnelle}

Cette dimension est liée à la représentation du monde physique. Deux catégories de descripteurs sont utilisées pour la caractériser : la facette de connaissance associée à l'acte identifié (souvent acte de langage) et les caractéristiques de cette facette.

Nous nous appuyons sur la définition donnée par Minstrell (1992) et Galili et Hazan (2000). Une facette est un énoncé mettant en jeu un élément de connaissance, de raisonnement ou de stratégie qui peut être correct ou incorrect du point de vue du savoir enseigné et par exemple relever alors de pratiques quotidiennes. Une facette est construite par le chercheur et sert de référence pour analyser le discours de la classe (professeur ou élève). Un élément de ce discours relève d'une facette quand le chercheur considère qu'il a le même sens que l'énoncé de la facette. Il y a donc un catalogue de facettes pour chaque corpus. 
Dans cette dimension représentationnelle nous avons également visé à caractériser :

- comment la facette se situe par rapport à la modélisation : relève-t-elle du monde de la théorie et du modèle ou de celui des observables (objets et événements) ou encore met-elle en relation ces deux mondes? (Tiberghien \& Malkoun, 2007);

- de quel type d'activité épistémique la facette relève, en particulier, est-elle associée à une activité d'interprétation, de prévision ou de description; il s'agit d'activités classiques en matière de modélisation;

- le type de langue quotidienne ou scientifique dont la facette relève. On considère ici des langues spécialisées ou encore des usages spécialisés des langues naturelles (Lerat, 1997).

Le tableau 3 récapitule l'ensemble de ces descripteurs.

\begin{tabular}{l|l|l}
\hline \multicolumn{2}{l}{ Facette } \\
\hline Nature épistémologique & Activité épistémique associée & Langue \\
\hline Monde des théories et modèles & Description & Scientifique \\
Monde des observables & Interprétation & Quotidienne \\
Mise en relation entre les deux mondes & Prévision & \\
\hline
\end{tabular}

Tableau 3 : trois descripteurs pour caractériser la dimension représentationnelle des actes et leurs modalités respectives associées

(II n'y a pas de correspondance ligne à ligne)

\subsection{Articulation des niveaux microscopique et mésocopique : l'épisode comme niveau intermédiaire}

Notre choix théorique nous a conduits à rendre compte sur le plan didactique des logiques des pratiques de classe, ou encore du sens de l'action des acteurs dans la classe avec des narrations (Sensevy, 2011). Ces narrations permettent de tenir compte de l'évolution temporelle des événements considérés comme pertinents dans le cadre théorique choisi.

La décomposition en actes du niveau microscopique ne conduit pas nécessairement à des descriptions narratives. Elle pourrait conduire à un dénombrement des actes et de leurs caractéristiques si le codage était fait systématiquement sur l'ensemble d'un corpus et permettrait ainsi une représentation numérique de la séance. Notre choix est différent, le codage en actes vise d'abord à nourrir la description narrative à partir d'événements observables.

Comme nous l'avons déjà précisé, l'épisode avait été initialement introduit à un niveau plus fin que le jeu et caractérisé avec les descripteurs mésoscopiques de la TACD (Venturini \& Tiberghien, 2012). Ici, nous considérons qu'il est à la bonne échelle pour articuler les niveaux mésoscopique et microscopique. Avec cette perspective, nous considérons que l'épisode peut être vu de deux manières différentes et complémentaires: - l'épisode est la plus petite unité de l'action conjointe examinée au regard de la transaction en cours associée au jeu. Ainsi, l'épisode a une unité constituée 
autour des éléments de savoir abordés et de la forme des interactions sociales. Au titre de plus petite unité de l'action conjointe, il peut être caractérisé à l'aide des descripteurs utilisés au niveau mésoscopique pour décrire l'action; - l'épisode peut aussi être caractériséà partir des actes analysés au niveau microscopique. Le découpage en épisodes peut se faire des points de vue mésoscopique (changement dans les genèses) et microscopique (discontinuité forte au niveau d'au moins une des trois dimensions des actes qui le constituent). De fait, comme nous le présentons ci-dessous, ces deux points de vue sont complémentaires, le découpage microscopique permettant généralement de conforter le découpage mésoscopique mais aussi parfois de l'affiner et de le réaménager à la marge.

\section{Méthodologie d'analyse de l'action didactique}

Précisons tout d'abord que nous ne décrivons ici qu'une partie de la méthode que nous utilisons dans l'analyse de l'action didactique, celle qui concerne directement les données vidéo. En effet, ces données ne sont pas les seules que nous utilisons habituellement dans nos analyses, il y a aussi par exemple les documents des élèves, les notes prises au vol lors de la séance, les textes institutionnels, les plans de classe ou des entretiens avec l'enseignant ou des élèves.

La (ou les) séance(s) est (sont) transcrite(s). Les propos des divers locuteurs sont pris en compte ainsi que certains gestes, en particulier ceux liés à la progression du savoir dans la classe. Dans les tableaux présentés ci-dessous nous avons rajouté quelques ponctuations de l'écrit pour faciliter la compréhension.

Chaque séance est aussi structurée au niveau mésoscopique, et notamment en jeux didactiques. Conformément aux concepts portés par la TACD, nous interprétons l'action didactique pour identifier en son sein des transactions successives, chacune marquée par un contrat, un milieu et un enjeu de savoir et chacune associée à un jeu. Ainsi, nous repérons par exemple, les moments où le milieu change de manière importante, où un savoir est institutionnalisé et où le contrat évolue, où une nouvelle question est posée dont la solution est liée à un nouveau savoir dans la classe, où l'enseignant énonce aux élèves l'enjeu de l'activité à venir.

Nous repérons ensuite au sein de chaque jeu des épisodes identifiés comme étant les plus petites unités transactionnelles disposant d'une (relative) autonomie et constitutives de la transaction. La délimitation des épisodes peut être remise en question à la suite de l'analyse microscopique.

Au sein des épisodes, nous identifions chaque acte verbal et non verbal, que nous caractérisons à l'aide des différents descripteurs relatifs aux trois dimensions pragmatique, interactionnelle et représentationnelle. Ces caractéristiques sont regroupées au sein d'un tableau identique à celui présenté dans les exemples illustrant la mise en œuvre de notre méthode d'analyse.

Cette caractérisation des différents actes constituant l'épisode permet d'abord de vérifier la pertinence des découpages précédents. Au changement d'épisode, 
certaines des caractéristiques des actes qui précèdent et qui succèdent la séparation entre les deux épisodes doivent évoluer significativement. Le sens et la caractérisation d'un épisode sont inférés à partir des caractéristiques de ses composantes (les actes au niveau microscopique) et de son contexte (notamment les jeux au niveau mésoscopique). En effet, selon Lemke (2000, p. 277) dont nous reprenons l'idée, pour comprendre ce qu'il se passe à une échelle de temps particulière, il faut au moins tenir compte des processus se déroulant aux niveaux immédiatement supérieur et immédiatement inférieur. Ces éléments permettent de construire une description interprétative et principalement chronologique de l'épisode (narration), traduisant la vie de la classe du point de vue transactionnel, c'est-à-dire du point de vue du savoir et des interactions qu'il suscite.

Ces narrations étant réalisées pour tous les épisodes du jeu, celui-ci fait lui-même l'objet d'une description interprétative selon les mêmes principes, à partir des caractéristiques des différents épisodes qui le constituent et d'informations issues des niveaux supérieurs (session, séquence, etc.). Cette description interprétative (narration) est fondée sur la métaphore du jeu. L'analyse des différents événements constitutifs de la narration permet d'associer à certains d'entre eux des modalités particulières des concepts caractérisant le niveau mésoscopique, notamment ici les genèses, certains des types d'action (définition, dévolution, régulation et institutionnalisation), ainsi que des éléments du contrat didactique. Notons que la description du jeu n'est pas la somme exacte des descriptions de chaque épisode, et qu'elle peut évoluer a posteriori en fonction des éléments importants dans les jeux suivants.

Les caractéristiques dominantes des différents jeux et particulièrement celles qui sont récurrentes, permettent enfin de qualifier les pratiques mises en œuvre au cours de la séance ou de la séquence.

Nous proposons dans la figure 1 une synthèse de la méthodologie exposée dans les paragraphes précédents.

\section{Exemples de mise en œuvre}

Les deux exemples que nous présentons ont été choisis dans des corpus déjà étudiés. Il s'agit de deux classes, en quatrième (autour de 14 ans) et en seconde (autour de 16 ans), avec des professeurs ayant une expérience d'enseignement différente. Alors que le professeur de quatrième est débutant, le professeur de seconde a une très longue expérience et a participé à un groupe de recherche-développement; on peut ainsi penser que la mise en œuvre du savoir, et donc le contrat ne seront pas les mêmes. La différence essentielle entre ces exemples porte sur les acteurs en jeu. Alors que le premier exemple est relatif à un travail en classe entière, dans le deuxième exemple, il s'agit d'un travail en petits groupes. Ceci permet de tester la robustesse des actes choisis dans des situations interactionnelles très différentes.

Nous ne détaillerons que le premier exemple, du fait de la limitation de la longueur de l'article. 


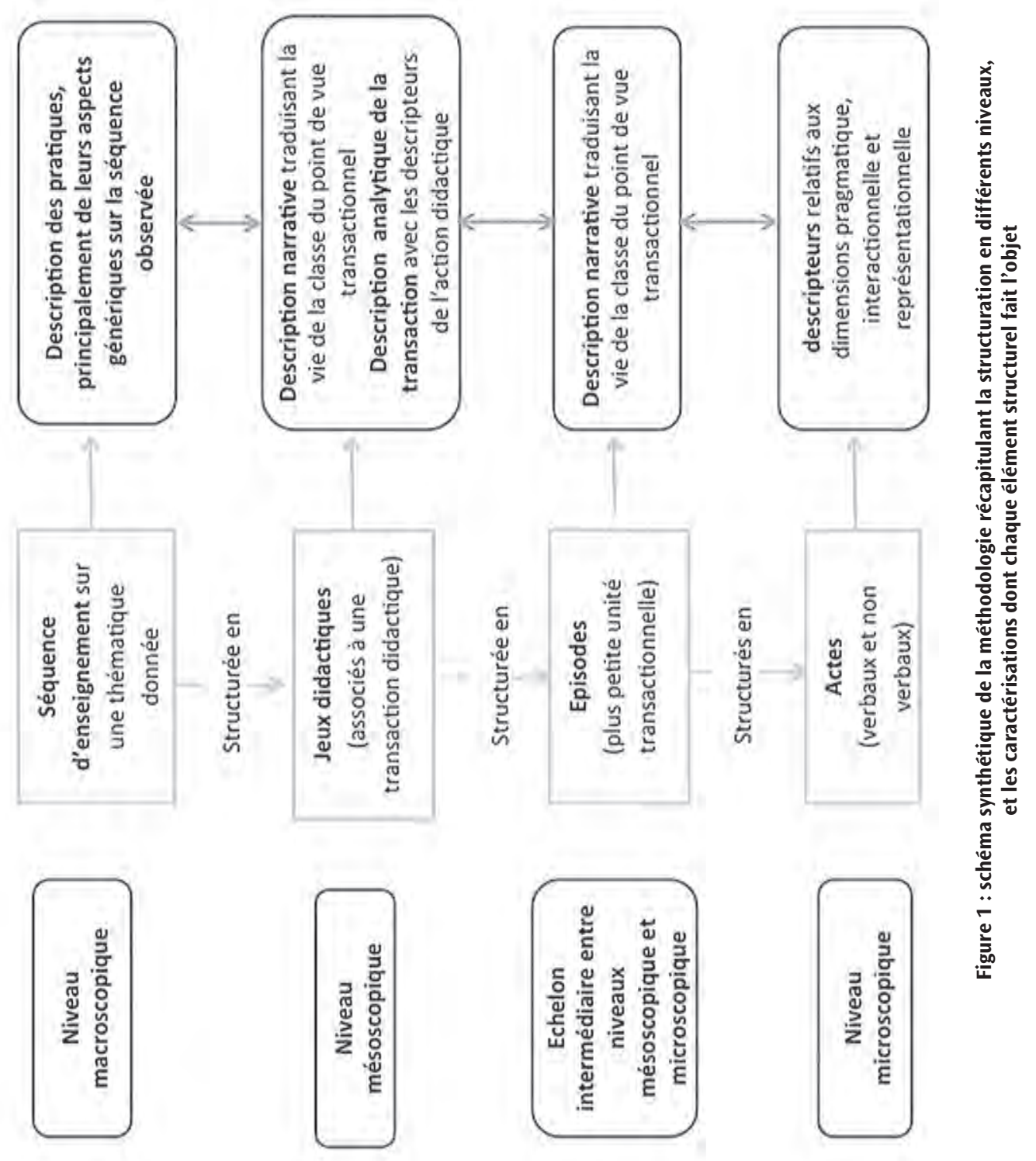




\subsection{Premier exemple : le démarrage d'une démarche d'investigation}

\subsubsection{Le contexte}

Le premier exemple de mise en œuvre de la méthode décrite précédemment est extrait d'une étude de cas concernant l'enseignement de la loi des tensions dans un circuit série en $8^{e}$ année dans une classe ordinaire d'un collège français (classe de $4^{e}$ pour la France). Le jeune professeur observé avait aussi pour objectif de mettre en œuvre une démarche d'investigation, conformément aux exigences curriculaires.

Ainsi, les élèves avaient à résoudre la situation problématique suivante pour établir la loi des tensions : " un garçon a construit une cabane dans laquelle il voulait passer la nuit. Effrayé par l'obscurité, il a voulu l'éclairer. Il a trouvé dans le garage de son père 5 ampoules dont le culot indiquait $0.1 \mathrm{~A}-3.5 \mathrm{~V}$ et 5 autres ampoules portant l'indication $0,2 \mathrm{~A}-6 \mathrm{~V}$, une batterie de 12 volts et des fils électriques. Il a pris le tout dans sa cabane, mis tous les composants les uns à la suite des autres, mais les lampes n'éclairaient pas". Le professeur a demandé alors aux élèves d'aider leur camarade pour parvenir à éclairer sa cabane.

Situons la partie dont nous présentons l'analyse. Il s'agit du $3^{\mathrm{e}}$ jeu de la séance, choisi parce qu'il est bref et donc facile à utiliser dans le contexte de cet article. L'enjeu des deux premiers jeux était respectivement, d'une part de rappeler la définition de la tension nominale et la procédure de mesure d'une tension aux bornes d'un dipôle, d'autre part d'identifier les éléments scientifiques inclus dans la description de la situation précédente. Professeur et élèves ont gagné à ces deux jeux, terminés à la minute 6:07. Le jeu suivant (jeu 3) a pour enjeu de proposer une solution/explication à la situation problématique proposée. Il s'agit d'une situation collective et nous détaillons l'analyse d'une partie du premier épisode du jeu. L'extrait proposé vient juste après les premiers tours de parole de cet épisode au cours desquels l'enseignant définit le jeu d'une manière qui peut être ambiguë pour les élèves leur demandant de "lui proposer une solution, une explication" relative à la situation proposée.

\subsubsection{Caractérisation de quelques actes selon les trois dimensions, pragmatique, interactionnelle et représentationnelle}

En référence au tableau 4, nous décrivons les caractéristiques de chacun des actes analysés. Le repérage se fait par rapport au numéro de ligne correspondant dans ce tableau rapportant la transcription.

Ligne (Li.) 4 : cette ligne comporte un seul acte (langagier), commencer à faire une proposition. Celle-ci est faite par une élève, en réponse à la question du professeur mentionnée plus haut. Nous ne voyons pas d'intention particulière dans ces propos, en dehors de celle de répondre à la question de l'enseignant. La question du professeur étant ouverte et sans indice particulier, nous considérons que cet élève apporte là une contribution étendue au thème discuté, que l'on peut formaliser par la facette "si l'ampoule brille mal, la pile n'est pas assez puissante". Cette facette qui s'étend 
sur deux lignes (4 et 6) est produite par une interprétation de la réalité décrite dans la situation en mettant en relation les différents observables et une théorie qui ici reste implicite, relevant des pratiques quotidiennes.

Li. 5 : le professeur, en poursuivant l'échange après la réponse de l'élève, répète sa proposition de manière neutre et sans rien apporter de nouveau, la note au tableau.

Li. 6 : l'élève termine la proposition qu'il fait en réponse à la question initiale du professeur. Cette proposition a sur le plan représentationnel, les mêmes caractéristiques que celle de la ligne 4 .

Li. 7 : cette ligne comporte plusieurs actes. Le professeur poursuivant l'échange à la suite du complément de réponse apporté par l'élève à la ligne précédente accepte sa proposition, qu'il évalue positivement. Toujours en poursuivant l'échange précédent, il demande ensuite une explication aux élèves à propos de la conclusion tirée, la partie "théorique" de l'interprétation menée étant implicite dans les propos. Nous interprétons cette demande d'explication comme une volonté de l'enseignant d'orienter les propos vers le monde des théories et modèles afin de les rapprocher d'une formalisation sur les tensions à laquelle il doit aboutir s'il souhaite enseigner la loi des tensions. Enfin, l'enseignant demande une autre proposition et initie ainsi une nouvelle série d'échanges par une question ouverte.

Li. 8 : un élève complète la proposition précédente en la prolongeant, en réponse à la question du professeur. Tirant la conséquence pratique de la proposition précédente, nous considérons la réponse de cet élève comme une contribution limitée au thème. En effet la facette correspondante (si la pile n'est pas assez puissante, mettre deux piles) met en relation les théories quotidiennes et l'univers des objets et les événements.

Li. 9 : le professeur poursuit l'échange entamé à la suite de sa question (Li. 7) en jugeant que les propositions faites sont des solutions. Cette déclaration intermédiaire lui permet de fonder le refus de ces propositions qu'il évalue négativement de manière implicite. Le professeur demande alors une nouvelle proposition de nature interprétative, qu'il reformule pour donner ainsi quelques indices sur ces attentes. Nous considérons que l'intention du professeur est à nouveau d'orienter les élèves vers l'univers des théories et des modèles, pour les mêmes raisons que sa précédente tentative en ce sens. 


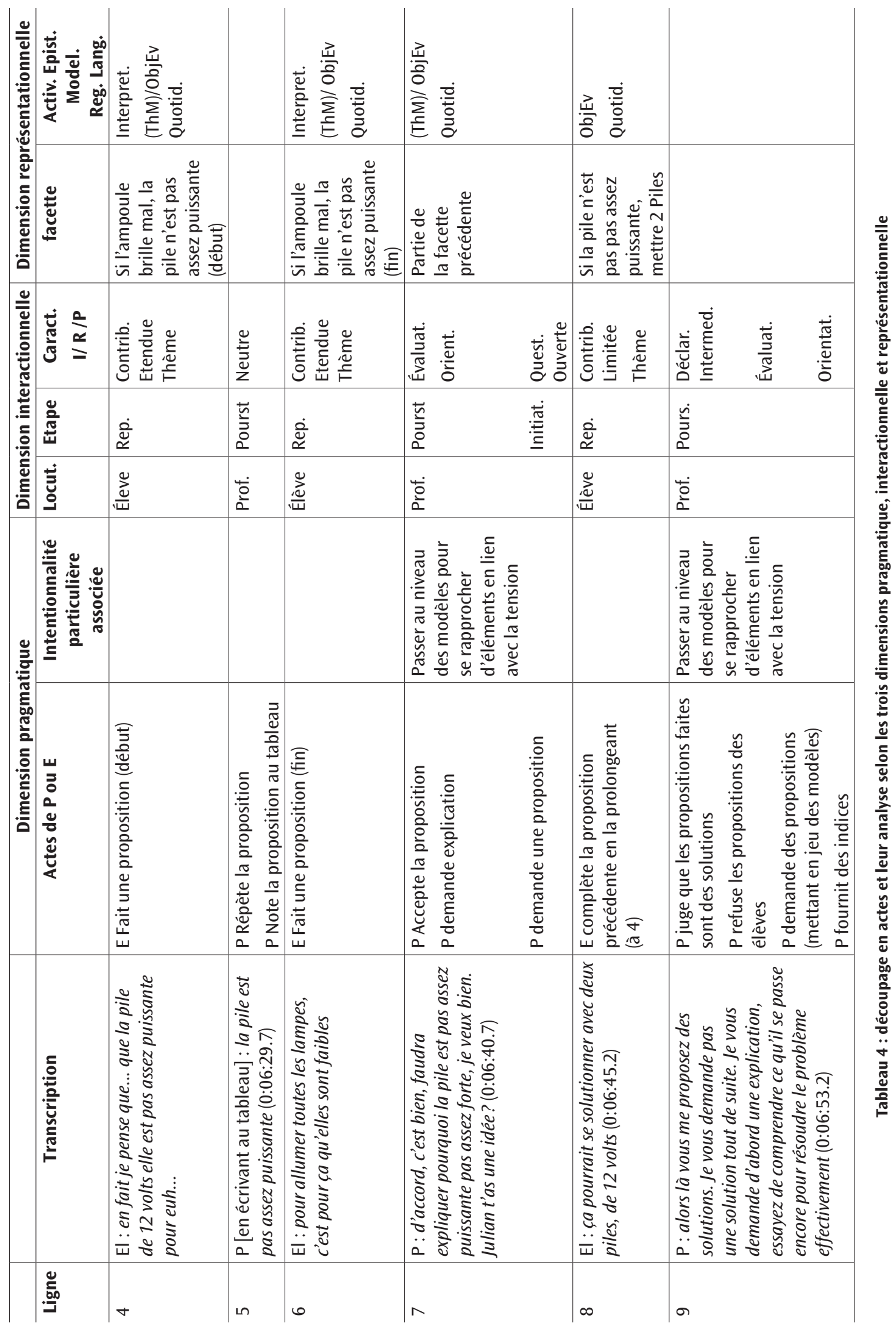




\subsubsection{Construction de la narration d'épisodes}

L'analyse microscopique précédente permet de réaliser une description narrative du déroulement de chacun des épisodes. Ainsi on peut narrer l'extrait précédent en s'appuyant sur l'analyse microscopique : Les élèves proposent dans des termes du langage quotidien des solutions fondées sur une relation causale simple (la pile n'est pas assez puissante - il faut mettre deux piles). Le professeur accepte leurs propositions sans les exploiter mais leur demande de proposer non plus une solution mais une explication à ce qui est observé.

Chaque épisode fait ainsi l'objet de ce type de description. Comme nous l'avons déjà évoqué précédemment, la description narrative associée à chaque épisode contribue à construire la description narrative du jeu. Celle-ci, n'est pas forcément la simple concaténation des descriptions de chaque épisode; selon l'importance que chacun d'entre eux a dans l'avancée du savoir par exemple l'introduction de nouvelles notions, nouveaux raisonnements, ou au contraire, l'abandon ultérieur de certains d'entre eux, il est possible que certains éléments de la description de chaque épisode soient abandonnés, ou au contraire mis plus en avant.

\subsubsection{Narration du jeu et sa caractérisation avec les descripteurs mésoscopiques}

Nous proposons ci-après la description narrative du jeu 3 construite à partir de la description narrative de l'épisode présenté ci-dessus et de celles des deux épisodes que nous ne fournissons pas. Nous avons rajouté entre parenthèses et en italique les descripteurs mésoscopiques que nous avons associés pour donner à voir au lecteur l'articulation entre description narrative de l'avancée du savoir et caractérisation conceptualisée du processus correspondant, qui figure après la description narrative du jeu.

Le professeur formule une demande à la fois de solution et d'explication à la situation problématique présentée préalablement, ce qui la rend ambiguë (définition du jeu). Les élèves proposent immédiatement des solutions (dévolution sur des aspects empiriques, liens avec des éléments pérennes $d u$ contrat) fondées sur une relation causale simple (la pile n'est pas assez puissante - il faut mettre deux piles) dans des termes du langage quotidien (genèse implicite ${ }^{7}$ du savoir). Le professeur accepte et note ces propositions au tableau (genèse du milieu) sans les exploiter (genèse des responsabilités) mais leur demande de proposer non plus une solution mais une explication à ce qui est observé (tentative de régulation pour orienter les élèves vers une interprétation de la situation). Cette tentative avorte, un élève faisant

7 Le chercheur peut considérer que l'idée de mettre deux piles dans le circuit qui relève de pratiques quotidiennes contribue à une avancée du savoir dans la classe, certes très modeste au regard de la loi des tensions dans un circuit série qu'il s'agit d'établir. Les propos correspondants relèvent à ce titre de la genèse du savoir. Toutefois, cette proposition, comme toutes les autres faites par les élèves n'est pas identifiable en tant que telle dans la classe, si bien que l'évolution du savoir reste dans l'implicite aux yeux des élèves. C'est la raison pour laquelle nous avons caractérisé cette proposition comme relevant d'une genèse implicite des savoirs. 
le même type de proposition causale impliquant le monde des objets et le langage du quotidien (enlever des ampoules) (genèse implicite du savoir; dévolution sur aspects empiriques). Cette proposition, comme toutes les précédentes, est acceptée sans discussion et notée au tableau (genèse du milieu, genèse des responsabilités). Une élève fait alors une proposition hypothétique d'interprétation conceptuelle de la situation selon laquelle "les volts de la pile" seraient inférieurs à la "somme des volts des ampoules" (genèse implicite du savoir). Le professeur accepte cette proposition sans commentaire et l'écrit au tableau (genèse du milieu, genèse des responsabilités), puis conclut en jugeant positivement l'ensemble des propositions faites par les élèves.

Les éléments complémentaires qui accompagnent la narration précédente permettent de la relier à la caractérisation correspondante ci-dessous :

- Les élèves assurent l'évolution du milieu au cours du jeu : la dévolution s'opère d'abord sur des aspects empiriques en lien avec des raisonnements causaux (simples) qui restent implicites, puis à la suite d'une régulation explicite du professeur exigeant non plus des solutions mais des explications (évolution de l'enjeu du jeu), la dévolution (qui semble ne plus concerner alors qu'une seule élève) s'opère sur des aspects conceptuels exprimés de manière relationnelle et hypothétique;

- Ce basculement s'accompagne aussi d'un changement du type de langue, passant du quotidien au scientifique. Probablement la dévolution est-elle facilitée par des éléments pérennes du contrat didactique permettant aux élèves de faire des propositions en toute confiance. La genèse du milieu se développe donc au cours du jeu, au rythme des propositions des élèves. Du point de vue du chercheur, les premières propositions des élèves peuvent être vues comme donnant une première intelligibilité de la situation et donc contribuer à une certaine avancée des savoirs. La dernière proposition quant à elle est tout à fait significative de ce point de vue. Mais ces propositions ne faisant l'objet d'aucun traitement particulier dans la classe, les avancées ne sont pas visibles des élèves;

- Enfin, si les élèves assurent l'évolution du milieu à l'aide de leurs propositions notées au tableau, le professeur ne leur demande pas d'en assumer la responsabilité. En effet, le professeur sollicite les élèves et accepte toutes les propositions telles quelles, sans en exploiter une seule, et aucune ne fait l'objet d'un débat ou d'une argumentation et encore moins d'une institutionnalisation. Aussi lui non plus n'exerce-t-il pas réellement de responsabilité vis-à-vis des savoirs en jeu, ce qui conduit à une topogenèse très particulière et à des échanges à la valence épistémique faible.

\subsection{Deuxième exemple : appliquer pour la première fois un modèle juste enseigné (travail en petits groupes)}

\subsubsection{Le contexte}

Le second exemple est extrait d'une étude portant sur l'enseignement de la mécanique dans l'ancien programme de seconde et plus particulièrement sur les sept premières séances relatives à la dynamique. Dans la classe observée, le professeur 
utilise une séquence élaborée par un groupe d'enseignants et de chercheurs auquel elle participe. L'extrait analysé est issu de la première séance relative à l'étude des interactions avec le diagramme système-interaction. L'exemple choisi correspond à un travail en petit groupe de deux élèves lors d'une activité faite à la fin de la séance. Elle consiste à tracer le diagramme système-interactions : "à l'aide du modèle des interactions, construire le diagramme système-interactions d'un objet posé sur une table (question a) puis d'une table sur laquelle est posé un objet (question b)".

Les deux élèves (deux filles) filmées commencent à réaliser l'exercice avant que le professeur n'ait fini de lire à haute voix l'énoncé. Nous donnons ci-dessous quelques échanges entre les deux élèves pour illustrer les points essentiels de l'analyse (voir tableau 5).

\subsubsection{Caractérisation de quelques actes selon les trois dimensions, pragmatique, interactionnelle et représentationnelle.}

En référence au tableau 5 nous décrivons les caractéristiques de chacun des actes analysés.

Dans la ligne 1, E1 oriente clairement l'action du binôme vers la reprise de l'activité proposée, ce à quoi E2 souscrit en enchaînant directement par une proposition (Li. 2). E1 (Li. 3) propose alors de limiter dans le diagramme les interactions à celles existant entre l'objet et la table sur laquelle ce dernier est posé, tout en s'interrogeant sur la pertinence d'un élément de savoir, l'attraction de la Terre, que sa proposition exclut de fait. Dans les deux cas, les facettes concernent une description scientifique du monde des objets et des événements.

En revanche, E2 dans la ligne 4, fait une contre-proposition, affirmant avec certitude qu'il faut prendre en compte la Terre dans les interactions, sans pour autant l'argumenter (contribution limitée au thème discuté). Non convaincue, E1 poursuit son interrogation (Li. 5) en demandant si cette contre-proposition est aussi valide sur la situation de la question (a) mettant en jeu un objet posé sur une table. E2 confirme sa proposition et fournit un argument d'une expérience de pensée mettant en jeu un raisonnement par l'absurde sur les objets considérés (Li. 6). E2 contribue ainsi de manière étendue au thème en cours, ce qui conduit probablement E1, dans la poursuite de l'échange, à déclarer que la Terre agit bien sur l'objet posé sur la table (Li. 7). 


\begin{tabular}{|c|c|c|c|c|}
\hline & & D pragmatique & D interactionnelle & D représentationnelle \\
\hline & Transcription & Actes P, E1, E2 & $\begin{array}{l}\text { (Étape) Car. } \\
\text { I/R/P }\end{array}$ & $\begin{array}{l}\text { (Facette) } \\
\text { Act. Epist. Model. } \\
\text { Reg. Lang. }\end{array}$ \\
\hline 1 & $\begin{array}{l}\text { E1 : bon alors objet } \\
(0: 41: 36.5)\end{array}$ & $\begin{array}{l}\text { E1 invite à } \\
\text { reprendre l'activité } \\
\text { en commun }\end{array}$ & (I) Injonc. & \\
\hline 2 & $\begin{array}{l}\text { E2 : alors objet } \\
\text { table }\end{array}$ & $\begin{array}{l}\text { E2 reprend l'activité } \\
\text { E2 fait proposition }\end{array}$ & Réponse & $\begin{array}{l}\left(1^{*}\right) \text { Descr } \\
\text { ObjEv }\end{array}$ \\
\hline 3 & $\begin{array}{l}\text { E1 : par contre y a } \\
\text { pas d'autre chose } \\
\text { quoi elle est sur } \\
\text { la table c'est tout } \\
\text { elle est pas attirée } \\
\text { par la Terre si (?) } \\
(0: 41: 43.0)\end{array}$ & $\begin{array}{l}\text { E1 fait une } \\
\text { proposition } \\
\text { E1 demande un } \\
\text { accord sur sa } \\
\text { proposition }\end{array}$ & $\begin{array}{l}\text { (I) } \\
\text { Quest. Fermée }\end{array}$ & $\begin{array}{l}\left(2^{*}\right) \text { Descr Interrog. } \\
\text { ThM/ObjEv } \\
\text { Sci }\end{array}$ \\
\hline 4 & $\begin{array}{l}\text { E2 : heu celui-là si } \\
(0: 41: 45.7)\end{array}$ & $\begin{array}{l}\text { E2 fait une } \\
\text { contre-proposition }\end{array}$ & $\begin{array}{l}\text { (R) Contrib. Limitée } \\
\text { Thème }\end{array}$ & $\left(3^{*}\right)$ \\
\hline 5 & $\begin{array}{l}\text { E1 : pour le a aussi? } \\
(0: 41: 47.9)\end{array}$ & $\begin{array}{l}\text { E1 demande } \\
\text { confirmation }\end{array}$ & $\begin{array}{l}\text { (I) Quest. } \\
\text { Fermée }\end{array}$ & (Idem 2) \\
\hline 6 & $\begin{array}{l}\text { E2 : ben ouais un } \\
\text { objet s'il est posé si } \\
\text { la Terre n'agissait } \\
\text { pas sur lui il serait } \\
\text { en l'air il volerait } \\
(0: 41: 56.3)\end{array}$ & $\begin{array}{l}\text { E2 confirme sa } \\
\text { proposition } \\
\text { E2 justifie sa } \\
\text { proposition }\end{array}$ & $\begin{array}{l}\text { (R) Contrib. } \\
\text { Etend. } \\
\text { Thème }\end{array}$ & $\begin{array}{l}\text { (4*) Descrip. } \\
\text { (en pensée) } \\
\text { ObjEv } \\
\text { Sci }\end{array}$ \\
\hline 7 & $\begin{array}{l}\text { E1: ah ouais donc } \\
\text { on a Terre et on } \\
\text { a quoi d'autre } \\
(0: 42: 00.0)\end{array}$ & $\begin{array}{l}\text { E1 accepte la } \\
\text { proposition } \\
\text { E1 demande } \\
\text { une proposition } \\
\text { complémentaire }\end{array}$ & $\begin{array}{l}\text { (P)Déclar. (I)Interm. } \\
\text { Quest. } \\
\text { Ouvert. }\end{array}$ & $\begin{array}{l}\text { (idem 3) Interpret. } \\
\text { ThM/OE, } \\
\text { Sci. }\end{array}$ \\
\hline
\end{tabular}

Tableau 5 : découpage en actes et leur analyse selon les trois dimensions pragmatique, interactionnelle et représentationnelle

Les rubriques de ce tableau sont limitées pour des raisons d'espace en comparaison de celles du tableau 4. Il manque notamment les facettes indiquées ci-après : $1^{*}$ : la table interagit avec le sol ; $2^{*}: y$-a-t-il toujours une interaction entre un objet et la Terre?; $3^{*}$ : la Terre agit toujours sur les objets; $4^{*}$ : si la Terre n'agissait pas sur les objets, ils voleraient. (E1 = Élève 1; E2 = Élève $2 ; \mathrm{I}=$ Initiation, $\mathbf{R}=$ Réponse, $\mathrm{P}=$ Poursuite)

L'analyse au niveau microscopique de cet extrait illustre l'importance des propositions dans les actes qui impliquent du savoir, soit en l'affirmant soit en l'interrogeant : ces actes manifestent la stratégie des élèves pour "jouer au jeu ". Comme dans l'extrait précédent, nous avons construit une narration des épisodes analysés qui montre que les éléments de savoir effectivement en jeu sont ceux visés par le professeur : pour ces épisodes, il y a dévolution, et les élèves traitent du savoir visé et en assurent la responsabilité. 
Le savoir avance non pas en termes de nouveaux éléments de savoir mais en termes de compréhension (plus approfondie) des éléments de savoir déjà introduits conformément à la nature de l'activité proposée aux élèves. D'ailleurs les propositions des élèves relèvent toutes de la langue scientifique, même si elles sont erronées par moment.

\section{Discussion des choix à propos de l'approche microscopique et de son rôle}

Cette discussion aborde sur différents plans le rôle joué par les analyses microscopiques dans les analyses de pratiques, leur intérêt et leurs limites.

Tout d'abord nous avons choisi d'emprunter à la linguistique pragmatique l'analyse en actes aussi bien verbaux que non verbaux, en reprenant l'idée de nouage entre action et communication caractérisé par les trois dimensions représentationnelle, pragmatique et interactionnelle de Charaudeau (2004). Ce choix nous a permis de rendre compte du rapport ternaire élève-professeur-savoir, à partir des productions verbales et partiellement non verbales du professeur et des élèves en prenant le point de vue didactique. Ainsi nous avons caractérisé les actes en prenant une perspective didactique où les actes relatifs aux propositions sont essentiels (voir tableau 1). Il faut noter que nos catégories microscopiques visent à être pertinentes pour des situations de classe différentes, comme le montrent les deux exemples choisis, l'un en classe entière et l'autre au sein d'un petit groupe.

Cette analyse microscopique en actes nous permet d'aller à la plus petite unité gardant un sens du point de vue de la transaction didactique. Ce point de vue commun des analyses permet des allers-retours pour construire le sens des actions. Ainsi un acte ne prend son sens que s'il est situé dans un empan plus large comme les narrations descriptives utilisées pour les niveaux supérieurs. Le sens d'un événement de l'ordre de la seconde ne peut se construire qu'au regard d'un ensemble d'autres événements ayant eu lieu à d'autres moments et sur des durées différentes. Mais le lien entre les niveaux n'est pas direct. Les actions didactiques du niveau mésoscopique comme la définition, la dévolution, la régulation et l'institutionnalisation, peuvent être mises en œuvre par des actes très différents (voir par exemple TIMSS video 1999 mené dans 5 pays, Roth et al., 2006). Il n'y a pas de relation biunivoque entre un acte et chacune de ces actions.

Les narrations des épisodes et des jeux sont nourries par l'analyse microscopique et inversement peuvent permettre de préciser les analyses en actes (cf. le point de vue de Lemke rapporté plus haut sur la nécessité de va-et-vient entre plusieurs échelles temporelles pour comprendre les activités complexes).

Ainsi cette articulation entre les niveaux permet d'enrichir la description des pratiques de classe d'une nouvelle "couche". En nous situant dans les débats sur l'épaisseur des descriptions en sciences humaines et sociales (Descombes, 1998), nous avançons l'idée que la couche microscopique introduite ici permet de garder 
un lien plus fort avec la situation effective lorsqu'il s'agit de la décrire. Cette couche permet d'expliciter et de justifier certaines analyses mésoscocopiques comme le choix "d'épisodes remarquables", elle renforce le fondement des analyses aux échelles supérieures.

Nous avons mis en évidence l'emprunt à la linguistique pour le niveau microscopique, mais de fait il ne se limite pas à ce niveau. Le choix des descriptions narratives est aussi lié à la linguistique. Ces relations entre analyse didactique et linguistique ne sont pas surprenantes puisque le langage joue un rôle crucial dans les pratiques de classe et dans les données récoltées et il n'est bien sûr pas "transparent".

Notre travail ayant porté sur le cas de l'enseignement de la physique dans des situations spécifiques, la question de son utilisation pour d'autres situations et contenus d'enseignement se pose. II nous semble que si le choix des actes avec leurs dimensions pragmatiques, interactionnelles et représentationnelles n'est pas lié à un contenu spécifique, les types d'actes, et surtout les facettes de la dimension représentationnelle seraient à adapter.

\section{Conclusion}

Ce travail d'ordre théorique et méthodologique trouve son origine dans une étude antérieure sur l'analyse des pratiques de classe principalement à partir de données vidéo. La question de l'utilisation des descripteurs issus de la TACD pour une analyse à l'échelle d'épisodes d'une ou deux minutes avait été soulevée ainsi que celle des liens que cette analyse entretiendrait avec des événements constitutifs de ces épisodes, de l'ordre de la seconde. Ces descripteurs sont constitués d'une part des différentes genèses (évolution du savoir, des responsabilités vis-à-vis du savoir et du milieu utilisé pour construire de nouvelles idées et donc contribuer au savoir) et d'autre part des types d'action du professeur, la définition, la dévolution, la régulation et l'institutionnalisation. Au départ de cette étude, et comme dans la précédente (Venturini \& Tiberghien, 2012), nous avons considéré que ces descripteurs sont pertinents au niveau mésoscopique (de l'ordre de la minute ou plus) et non au niveau microscopique (de l'ordre de quelques secondes) car leur signification est fondée sur la durée. Nous avons donc développé des descripteurs spécifiques de ce niveau dont cet article rend compte. Pour cela nous avons fait appel à des travaux menés sur l'interaction et l'analyse du discours par des chercheurs ayant une approche pragmatique. C'est ainsi que nous avons choisi, en référence aux actes de langage (Searle, 1969), l'acte comme unité microscopique, en ne limitant pas l'acte au verbal. Nous avons repris de Charaudeau (2004) les trois conceptions représentationnelle, pragmatique et interactionnelle qui permettent de nouer l'action et le langage pour caractériser les actes. Cet emprunt visait à construire un tout cohérent avec l'orientation actionnelle de la TACD, et cela s'est vérifié empiriquement. Cette cohérence empirique s'est manifestée par l'utilisation dans les dimensions représentationnelle et interactionnelle d'analyses développées dans des travaux antérieurs. Ainsi l'analyse du savoir en facettes et en niveaux de modélisation issue 
de travaux antérieurs a été appliquée quasi directement. Concernant l'aspect interactionnel, nous avons repris le pattern d'interaction "Initiation - Réponse - Poursuite" mis en évidence par Sinclair et Coulthard, retravaillé pour le cas de la réponse par Graesser et Person (1994), et pour le cas de la poursuite par des chercheurs comme Chin (2006), afin d'adapter ce pattern et sa caractérisation à des cas variés d'interaction. En particulier, nous l'avons exploité pour caractériser les interactions entre le professeur et un petit groupe d'élèves ou entre élèves dans le cas d'un travail individuel ou en petits groupes. Nous avons alors élaboré de manière inductive l'opérationnalisation de ce pattern. Si les dimensions interactionnelles et représentationnelles ont fait très majoritairement l'objet d'exploitation de travaux antérieurs, en revanche, la caractérisation de la dimension pragmatique a été essentiellement inductive. Malgré de nombreux travaux antérieurs cela a été en effet nécessaire pour que la catégorisation des actes identifiés soit cohérente avec notre perspective d'étudier l'avancée et la mise en œuvre du savoir par le professeur et les élèves. Ainsi, notre analyse microscopique, nourrie de travaux antérieurs et de développements inductifs, a pu être menée sur des extraits vidéo de classe variés sans avoir à un moment ou un autre à remettre en cause nos choix. On aura d'ailleurs constaté que les extraits illustratifs choisis diffèrent sur plusieurs aspects, le niveau d'enseignement, l'expérience des professeurs, le type de situations de classe où les productions du professeur et des élèves sont publiques dans le premier exemple et privées au sein d'un petit groupe dans le deuxième exemple, et donc présentent des enjeux de savoir différents.

Nous avons posé que la narration, présentée du point de vue des relations savoir-professeur-élèves, était essentielle pour rendre compte sur le plan didactique des pratiques de classe. Cela nous a conduits à articuler les niveaux microscopique et mésoscopique par une narration, celle de l'épisode.

Cette narration est nourrie par l'analyse microscopique mais n'ignore pas des événements qui surviennent à d'autres échelles temporelles. Compte tenu du point de vue pris, elle inclut également l'évolution temporelle et la prise en charge du savoir par les acteurs, si bien qu'elle peut aussi être considérée comme une narration mésoscopique. La combinaison des narrations des différents épisodes (qui n'est pas leur simple concaténation) permet de construire la narration du jeu. L'épisode constitue donc ici un intermédiaire entre les niveaux microscopique et mésoscopique ; ainsi la narration du point de vue du développement du savoir dans la classe est un moyen permettant de les articuler. La narration du jeu incorpore les évolutions du savoir et la caractérisation des interactions associées, en leur donnant une signification sur une échelle temporelle plus conséquente dont les descripteurs de la TACD rendent compte.

Ainsi, l'analyse microscopique enrichit les narrations, en les ancrant de manière plus précise à des événements observés directement sur la vidéo, et renforce le lien données-interprétations.

Si cet aspect constitue selon nous une avancée dans les analyses de vidéos de classe en physique, cette étude présente aussi plusieurs limites; nous en proposons 
deux, qui constituent autant de perspectives possibles à moyen terme pour la prolonger. L'étude présentée porte sur le niveau microscopique et ses liens avec le mésoscopique, elle n'aborde pas les échelles macroscopiques sur une séquence de plusieurs semaines ni a fortiori les pratiques de classe sur plusieurs années. Dans ce cas, de nouveaux niveaux intermédiaires devront être construits, analysés et articulés pour relier des événements se déroulant à différentes échelles temporelles. Ainsi pourrait-on documenter comment des événements à des échelles très courtes contribuent à instaurer sur des durées d'un à trois mois un contrat didactique particulier dans la classe.

Une autre limite de l'étude présentée ici est relative au temps que le chercheur doit passer pour conduire ces analyses microscopiques sur de longues périodes temporelles. Cette durée risque d'aller au-delà de ce qu'il est possible de faire. II faut donc, si l'on juge nécessaire ce niveau microscopique d'analyse, aller vers une instrumentation permettant une aide substantielle au chercheur. Il s'agit d'un champ de recherche relativement nouveau en didactique.

Nous remercions un des relecteurs ainsi que Peter Griggs de l'UMR ICAR pour leurs commentaires fructueux sur nos emprunts à la linguistique.

Andrée Tiberghien andrée.tiberghien@ens-lyon.fr

Patrice Venturini Patrice Venturini@univ-tlse2.fr

\section{Bibliographie}

BLOOM B. S., ENGELHART M. D., FURST E. J., HILL W. H. \& KRATHWOHL D. R. (1956). Taxonomy of educational objectives: the classification of educational goals. Handbook I. New York : David McKay.

Charaudeau P. (2004). Comment le langage se noue à l'action dans un modèle socio-communicationnel du discours. De l'action au pouvoir. Cahiers de linguistique française, Les modèles du discours face au concept d'action. Actes du $9^{\mathrm{e}}$ colloque de Pragmatique de Genève et colloque Charles Bally, université de Genève, Genève, 26. En ligne :<http://www.patrick-charaudeau.com/Commentle-langage-se-noue-a-l,90.html $>$.

CHIN C. (2006). Classroom Interaction in Science: Teacher questioning and feedback to students' responses. International Journal of Science Education, vol. 28, $\mathrm{n}^{0} 11$, p. 1315-1346.

DESCOMBES V. (1998). La confusion des langues Enquête, 6, 35-54. En ligne : $<$ http://enquete.revues.org/1403>. doi:10.4000/ enquete.1403. 
FILLIETAZ L. (2004). Introduction. Cahiers de linguistique française, Les modèles du discours face au concept d'action. Actes du $9^{\mathrm{e}}$ colloque de Pragmatique de Genève et colloque Charles Bally, Université de Genève, Genève, 26. En ligne : <http://clf. unige.ch/display.php?numero $=26 \&$ idFichier $=72>$.

GALILI I. \& HAZAN A. (2000). The influence of an historically oriented course on students' content knowledge in optics evaluated by means of facets-schemes analysis. American Journal of Physics, vol. 68, supplement n 7, S3-S15.

GRAESSER A. C. \& PERSON N. K. (1994). Question Asking During Tutoring. American Educational Research Journal, vol. 31, nº1, p. 104-137.

KERBRAT-ORECCHIONI C. (1995). Où en sont les actes de langage? L'Information grammaticale, $\mathrm{n}^{\circ} 66$, p. 5-12.

LEMKE J. L. (2000). Across the Scales of Time: Artifacts, Activities, and Meanings in Ecosocial Systems. Mind, culture, and activity, vol. 7, nº 4, p. 273-290.

LEMKE J. L. (2001). The long and the short of it: comments on multiple timescale studies of human activities. The Journal of the Learning Sciences, vol. 10, $\mathrm{n}^{0} 1-2$, p. 17-26.

LERAT P. (1997). Approches linguistiques des langues spécialisées ASp, p. 15-18. En ligne: < http://asp.revues.org/2926>. doi:10.4000/asp.2926.

MINSTRELL J. (1992). Facets of students' knowledge and relevant instruction. In R. Duit, F. Goldberg \& H. Niedderer (éd.), Research in physics learning: Theoretical issues and empirical studies, Kiel : IPN, p. 110-128.

MORTIMER E. F. \& SCOTT P. H. (2003). Meaning Making in Secondary Science Classroom. Milton Keynes : Open University Press.

MORTIMER E. F., MASSICAME T., TIBERGHIEN A. \& BUTY C. (2007). Uma metodologia para caracterizar os gêneros de discurso como tipos de estratégias enunciativas nas aulas de ciências. In R. Nardi (éd.), A pesquisa em ensino deciências no Brasil: alguns recortes, São Paulo : Escrituras, p. 53-94.

ROTH K. J., DRUKER S. L., GARNIER H. E., LEMMENS M., CHEN C., KAWANAKA T., et al. (2006). Teaching Science in Five Countries: Results From the TIMSS 1999 Video Study Statistical Analysis Report (NCES 2006-011). Washington : U.S. Department of Education, National Center for Education Statistics/Washington, DC : U.S. Government Printing Office.

SCOTT P., MORTIMER E. \& AGUIAR 0. (2006). The tension between authoritative and dialogic discourse: a fundamental characteristic of meaning making interactions in high school science lessons. Science Education, nº 90, p. 605-631.

SEARLE J. R. (1969/1972). Les actes de langage. Paris : Hermann. 
SEARLE J. R. \& VANDERVEKEN D. (1985). Foundations of illocutionary logic. Cambridge : Cambridge University Press.

SENSEVY G. (2011). Le sens du savoir. Éléments pour une théorie de l'action conjointe en didactique. Bruxelles: De Boeck.

SENSEVY G. (2013). Milieu. Springer Encyclopedia of Science Education.

SINCLAIR J. M. H. \& COULTHARD R. M. (1975). Towards an analysis of discourse. London : Open University Press.

SINCLAIR J. M. H. \& COULTHARD R. M. (1992). Towards an analysis of discourse. In M. Coulthard (éd.), Advances in spoken discourse. London : Routledge, p. 1-34.

TIBERGHIEN A. \& MALKOUN L. (2007). Différenciation des pratiques d'enseignement et acquisitions des élèves du point de vue du savoir. Éducation et didactique, p. 29-54.

TIBERGHIEN A., MALKOUN L., BUTY C., SOUASSY N. \& MORTIMER E. (2007). Analyse des savoirs en jeu en classe de physique à différentes échelles de temps. In G. Sensevy \& A. Mercier (éd.), Agir ensemble : éléments de théorisation de l'action conjointe du professeur et des élèves. Rennes: Presses universitaires de Rennes, p. 93-122.

TIBERGHIEN A., MALKOUN L. \& SECK M. (2008). Analyse des pratiques de classes de physique : aspects théoriques et méthodologiques. Les Dossiers des sciences de l'éducation, nº 19, p. 61-79.

VENTURINI P. \& TIBERGHIEN A. (2012). Mise en œuvre de la démarche d'investigation dans le cadre des nouveaux programmes de sciences physiques et chimiques : étude de cas au collège. Revue française de pédagogie, $\mathrm{n}^{\circ} 180$, p. 95-120.

WORTHAM S. (2006). Learning Identity: The Joint Emergence of Social Identification and Academic Learning. Cambridge : Cambridge University Press. 